\title{
Significados atribuídos às complicações de estomia e pele periestoma em um serviço de referência na região do Cariri
}

\section{Meanings attributed to complications of ostomy and skin peristhectomy in a public reference service in the Cariri region}

\section{Significados atribuidos a las complicaciones de ostomía y piel periestomal en un servicio de referencia en la región de Cariri}

Yterfania Soares Feitosa1,2, Luis Rafael Leite Sampaio 1,2, Déborah Albuquerque Alves Moreira1, Francisco Antonio da Cruz Mendonça ${ }^{3}$, Maria Corina Amaral Viana ${ }^{1}$, Katia Pires Nascimento do Sacramento ${ }^{1}$, Fernanda Maria Silva ${ }^{1}$, Tatyelle Bezerra Carvalho ${ }^{1}$, Yara Lanne Santiago Galdino ${ }^{4}$

ORCID IDS

Feitosa YS (iD https://orcid.org/0000-0002-6021-7557

Sampaio LRL (iD https://orcid.org/0000-0003-1437-9421

Moreira DAA (D) https://orcid.org/0000-0002-2823-8681

Mendonça FAC (iD https://orcid.org/0000-0002-3957-7048

Viana MCA (D) https://orcid.org/0000-0002-6890-9400

Sacramento KPN (iD https://orcid.org/0000-0002-6609-3479

Silva FM (iD https://orcid.org/0000-0002-0504-9896

Carvalho TB (iD https://orcid.org/0000-0001-6515-7963

Galdino YLS (iD https://orcid.org/0000-0001-9242-1715

\section{COMO CITAR}

Feitosa YS, Sampaio LRL, Moreira DAA, Mendonça FAC, Viana MCA, Sacramento KPN, Silva FM, Carvalho TB, Galdino YLS. Significados atribuídos às complicações de estomia e pele periestoma em um serviço de referência na região do Cariri. ESTIMA, Braz. J. Enterostomal Ther., 16: e3918. https://doi.org/10.30886/estima.v16.651 PT

\section{RESUMO}

Objetivos: Apresentar os significados atribuídos por participantes ou seus cuidadores em relação às complicações da estomia e pele periestoma em um serviço de referência na região do Cariri. Métodos: Pesquisa descritiva, de natureza qualitativa, composta por 24 participantes com estomias intestinais. Os dados foram coletados no período de março e junho de 2018 no Serviço de Atenção à Saúde da Pessoa com Estomia localizado no município de Juazeiro do Norte, estado do Ceará. As entrevistas foram guiadas por um roteiro estruturado e gravadas por meio de um aplicativo de celular. Durante a análise dos dados, as respostas dos participantes foram transcritas na íntegra. Resultados: A partir do relato dos participantes, emergiram os seguintes significados para as complicações da estomia e pele periestoma: aspectos relacionados à qualidade dos equipamentos coletores, como tempo de permanência da bolsa, tipo de bolsa e adesividade; fragilidade no processo educativo; e efetividade dos procedimentos cirúrgicos. Conclusão: A prestação de assistência especializada se faz necessária para que as orientações dos profissionais estejam direcionadas para as necessidades individuais de cada participante, uma vez que a assistência em saúde se encontra pautada em um processo interpessoal centrado no cuidado integral do indivíduo e sua família.

DESCRITORES: Estomaterapia; Estomia; Cuidados de enfermagem.

\footnotetext{
'Universidade Regional do Cariri - Centro de Ciências Biológicas e da Saúde - Departamento de Enfermagem - Crato/CE - Brasil. 2Universidade de Fortaleza - Departamento de Enfermagem - Fortaleza/CE - Brasil.

${ }^{3}$ Centro Universitário Estácio do Ceará - Centro de Ciência da Saúde - Departamento de Enfermagem - Fortaleza/CE - Brasil. ${ }^{4}$ Prefeitura Municipal de Fortaleza - Secretaria Municipal de Saúde - Estratégia Saúde da Família - Fortaleza/CE - Brasil. Autor correspondente: Luis Rafael Leite Sampaio | Rua Coronel Antônio Luis, 1161 - Pimenta | CEP: 63105-000 - Crato/CE - Brasil | E-mail: rafael.sampaio@urca.br Recebido: Set 242018 | Aceito: Jan 132019
} 


\begin{abstract}
Objectives: To present the meanings attributed by participants or their caregivers in relation to complications of the ostomy and peristomal skin in a referral service in the Cariri region. Methods: Descriptive research, of a qualitative nature, composed of 24 participants with intestinal ostomies. The data were collected in the period of March and June of 2018 in the Service of Health Care of the Person with Ostomy located in the city of Juazeiro do Norte, state of Ceará. The interviews were guided by a structured script and recorded through a mobile app. During the data analysis, participants' answers were transcribed in its full. Results: From the report of the participants, the following meanings for the complications of the ostomy and peristomal skin emerged: aspects related to the quality of the collecting equipment, such as time of permanence of the bag, type of bag and adhesiveness; fragility in the educational process; and effectiveness of surgical procedures. Conclusion: The provision of specialized assistance is necessary so that the orientations of the professionals are directed to the individual needs of each participant, since health care is based on an interpersonal process centered on the integral care of the individual and his or her family.
\end{abstract}

DESCRIPTORS: Stomatherapy; Ostomy; Nursing care.

\title{
RESUMEN
}

Objetivos: Presentar los significados atribuidos por participantes o sus cuidadores con relación a las complicaciones de la ostomía y piel periestomal en un servicio de referencia en la región de Cariri. Métodos: Investigación descriptiva, de naturaleza cualitativa, compuesta por 24 participantes con ostomías intestinales. Los datos fueron recolectados en el período de marzo y junio del 2018 en el Servicio de Atención a la Salud de la Persona con Ostomía localizado en el municipio de Juazeiro do Norte, estado de Ceará. Las entrevistas fueron guiadas por un guión estructurado y grabadas por medio de una aplicación de celular. Durante el análisis de los datos, las respuestas de los participantes fueron transcritas íntegramente. Resultados: A partir del relato de los participantes, emergieron los siguientes significados para las complicaciones de la ostomía y piel periestomal: aspectos relacionados a la calidad de los equipos colectores, como tiempo de permanencia de la bolsa, tipo de bolsa y adhesividad; fragilidad en el proceso educativo; y efectividad de los procedimientos quirúrgicos. Conclusión: La prestación de asistencia especializada se hace necesaria para que las orientaciones de los profesionales estén dirigidas para las necesidades individuales de cada participante, una vez que la asistencia de salud se encuentra pautada en un proceso interpersonal centrado en el cuidado integral del individuo y de su familia.

DESCRIPTORES: Ostomaterapia; Ostomía; Cuidados de enfermería.

\section{INTRODUÇÃO}

Entende-se por estomia uma abertura produzida cirurgicamente para comunicar, de forma temporária ou permanente, um órgão oco com o ambiente externo através da pele ${ }^{1}$. Dependendo do segmento do órgão exteriorizado, as estomias podem receber diferentes denominações, como colostomias (cólon), ileostomias (segmento ileal), traqueostomias (traqueia), dentre outras.

Frente à variabilidade de estomias, merecem destaque as intestinais, visto que a maioria das complicações está relacionada a esse tipo de estomia, pois tanto o contato do efluente com a pele periestoma como a técnica cirúrgica utilizada podem causar danos que comprometem a qualidade de vida $(\mathrm{QV})$ da pessoa com estomia ${ }^{2}$.

Diversas são as causas relacionadas à necessidade de confecção de uma estomia intestinal, como traumas, doenças intestinais inflamatórias, prolapso retal, disfunção neurológica grave, atrofia vesical, neoplasias de colón, reto e bexiga ${ }^{3}$. Entretanto, as neoplasias constituem, atualmente, as causas mais comuns de indicação de estomias, sendo o câncer colorretal o agravo mais frequente para confecção da estomia definitivo ${ }^{4}$.

De acordo com o Instituto Nacional de Câncer, o câncer colorretal é a terceira neoplasia maligna mais comumente diagnosticada e a quarta principal causa de morte por câncer, representando 1,4 milhão de casos novos e quase $700 \mathrm{mil}$ óbitos em $2012^{5}$.

As complicações relacionadas às estomias podem ser classificadas em precoces (sangramento, edema, infecção, dermatite periestoma, retração, isquemia ou necrose de alça exteriorizada) ou tardias (estenose, obstrução, prolapso, hérnia paraestomal e fístulas) ${ }^{6}$.

Um estudo transversal retrospectivo realizado com 572 prontuários de pessoas com estomias atendidos na Associação dos Ostomizados do Rio Grande do Norte, no período de 1991 a 2015, evidenciou que a dermatite, o prolapso, a hérnia paraestomal e a retração da estomia foram as complicações mais frequentemente observadas naquela população ${ }^{7}$. 
As complicações podem, no entanto, ser evitadas com o planejamento local da confecção da estomia e com o uso de técnica cirúrgica adequada ${ }^{8}$. A demarcação da estomia consiste na determinação dos limites para sua confecção, podendo ser realizada pelo enfermeiro estomaterapeuta a fim de favorecer, durante $\mathrm{o}$ ato cirúrgico, a confecção de uma abertura em local adequado que permita a adaptação de equipamentos para coleta de efluentes com o mínimo de desconforto para o paciente9 .

O seguimento da padronização da localização da estomia reduz significativamente o índice das complicações, uma vez que situar a estomia em uma área que assegure a aderência do equipamento e que seja de fácil visualização para a pessoa constitui uma estratégia de prevenção de complicações como estenose, prolapso, retração e isquemia ${ }^{10}$.

A orientação multiprofissional, bem como apoio familiar, favorece a adaptação à nova situação de vida e melhora a postura em relação ao autocuidado. O processo educativo em saúde é um instrumento de socialização de conhecimento, de promoção da saúde e prevenção de doenças. Dentre os profissionais de saúde que estão sempre em busca de materiais educativos a fim de subsidiarem o processo do cuidar, os enfermeiros são constantemente desafiados a buscar opções que deem suporte para atuarem com pessoas, grupos e comunidades ${ }^{11}$.

Dessa forma, o interesse acerca dessa temática surgiu a partir das experiências vivenciadas pelos autores durante $o$ acompanhamento de pessoas com complicações em estomias intestinais e pele periestoma. Dada a relevância de se ampliarem as discussões acerca dessa problemática em função do impacto emocional, físico e socioeconômico causado por suas crescentes taxas de morbidade, sentiu-se, portanto, a necessidade de se avaliar quais os significados atribuídos pelas pessoas com estomias intestinais ou seus cuidadores acerca do surgimento dessas complicações.

Diante do exposto, o estudo tem por objetivo apresentar os significados atribuídos por participantes ou seus cuidadores em relação às complicações da estomia e pele periestoma em um serviço de referência na região do Cariri.

\section{MÉTODOS}

Trata-se de estudo descritivo com abordagem qualitativa desenvolvido a partir do projeto de dissertação apresentado ao Programa de Pós-Graduação do Mestrado Profissional em Tecnologia e Inovação em Enfermagem da Universidade de Fortaleza (UNIFOR).
Para pesquisa qualitativa, o enfoque interpretativista do mundo e da sociedade deve ser entendido segundo a perspectiva daqueles que o vivenciam, o que implica considerar que o objeto de pesquisa é compreendido como sendo construído socialmente. Dessa forma, a pesquisa qualitativa torna-se importante para o estudo da experiência vivida, dos longos e complexos processos de interação social ${ }^{12}$.

De acordo com o serviço de referência à pessoa com estomia, para a município de Juazeiro do Norte, estado do Ceará, há 73 indivíduos com estomias intestinais cadastrados no Programa de Atenção à Saúde da Pessoa Ostomizada da Secretaria Estadual de Saúde do Estado do Ceará. Desses, 25 não compareceram no período da coleta e seis não aceitaram participar do estudo, perfazendo o total de 42 participantes entrevistados: 24 com estomias e 18 cuidadores.

Durante os encontros, apresentaram-se, inicialmente, os objetivos da pesquisa e o Termo de Consentimento Livre e Esclarecido (TCLE). A coleta dos dados foi realizada por meio de entrevistas gravadas por um aplicativo de celular e com auxílio de um roteiro estruturado adaptado do Programa de Atenção à Saúde da Pessoa Ostomizada do Estado do Ceará que contemplou questões referentes às complicações nas estomias e pele periestoma. Após gravadas, as respostas dos entrevistados foram transcritas na íntegra para melhor serem analisadas e codificadas em ordem cronológica pela letra $\mathrm{E}$ para os participantes com estomias e $\mathrm{C}$ para os participantes cuidadores, seguidas de numeração arábica.

A maneira mais confiável de se preservar ou de se reproduzir com precisão o conteúdo da entrevista é por meio do registro das anotações ou com auxílio de um gravador, uma vez que os limites da memória humana não possibilitariam a retenção da totalidade da informação, levando a possíveis distorções decorrentes dos elementos subjetivos que se projetam durante a reprodução da entrevista ${ }^{12}$.

A análise dos dados se deu por meio da técnica de análise de conteúdo de Bardin, seguindo as etapas de pré-análise, exploração do material e tratamento dos resultados, inferência e interpretação. Entende-se por análise de conteúdo um conjunto de instrumentos metodológicos que visa a analisar diferentes formas de comunicação, verbal ou não verbal, obtidas por meio de entrevistas ou observação direta, cujo material extraído após análise minuciosa e exaustiva é classificado/separado em temas ou categorias ${ }^{13}$. 
A participação no estudo se deu de forma voluntária, mediante a assinatura do TCLE e com base na Resolução do Conselho Nacional de Saúde no 466/12, a qual "incorpora, sob a ótica do indivíduo e das coletividades, referenciais da bioética como, autonomia, não maleficência, beneficência, justiça e equidade"e afirma, em seu capítulo IV, que o respeito devido à dignidade humana exige que toda pesquisa se processe após consentimento livre e esclarecido dos sujeitos, indivíduos ou grupos que manifestem a sua anuência à participação na pesquisa.

Nesse sentido, garantiu-se aos participantes seu anonimato, sua privacidade, seu direito de autonomia referente à liberdade de participar ou não da pesquisa, bem como de desistir em qualquer etapa da sua realização. O projeto foi apreciado e aprovado pelo Comitê de Ética em Pesquisa da UNIFOR sob parecer 2.542.686 e CAAE 83103518.4.0000.5052.

Os autores declaram não haver conflitos de interesse políticos e/ou financeiros associados à provisão dos materiais utilizados no estudo, bem como nenhum tipo de financiamento para sua realização.

\section{RESULTADOS E DISCUSSÃO}

O discurso evidenciado a partir da análise dos participantes aponta para as causalidades atribuídas às complicações das estomias e pele periestoma.

Para alguns entrevistados, a situação clínica é oriunda do tempo de permanência da bolsa:

Fica avermelhado quando passa de 5 a 6 dias e fica coçando, aí, quando eu tiro a bolsa, faço a limpeza, aí volta ao normal. É, já fica irritado. Fica coçando e vermelho. (E42)

Entre as principais complicações na pele periestoma, a dermatite é a mais comum. Trata-se de um processo patológico envolvendo todas as lesões de pele ao redor da estomia manifestas por meio de sinais como eritema, rubor, dor, calor ou lesões primarias de pele ${ }^{14}$. Dessa forma, a estratégia de tratamento global para a maioria das complicações da pele periestoma após o diagnóstico correto é ajustar o equipamento coletor e adicionar terapia tópica para promover a cicatrização ${ }^{15}$. Assim, a escolha do equipamento coletor adequado pelo estomaterapeuta aliada ao ensino do autocuidado é fundamental para evitar o surgimento desse tipo de complicação ${ }^{16}$.
Como regra geral, o melhor equipamento coletor é o mais simples, confortável, prático, seguro, discreto, de menor custo e que se adapte bem à estomia e proteja a pele periestoma, cabendo, portanto, ao profissional, explicar à pessoa com estomia as vantagens e desvantagens de cada bolsa coletora conforme as características individuais e sua escolha pessoal ${ }^{1}$.

Para alguns participantes com estomia, o prolapso apresentado na estomia é decorrente do esforço físico:

Isso aqui foi o seguinte, foi o peso que eu peguei uma vez. Cometi um erro, eu não sabia, entendeu? Aí, eu carregando quatro estacas, trabalhando igual eu gosto de trabalhar mesmo, aí quando eu joguei as estacas senti uma dor, senti uma dor, passei uns 3 dias em casa, aí fui pro hospital, certo, através do peso que eu peguei. (E2, E5, E23)

O prolapso é um tipo de complicação tardia decorrente da exteriorização do segmento intestinal móvel que pode ser resultante de anomalias da estrutura abdominal ou da dificuldade para a confecção da estomia ${ }^{17}$. Trata-se de uma complicação pouco frequente nas ileostomias (6\%) e colostomias (5\%), geralmente relacionada a uma hérnia paraestomal, obesidade e inserção de estomias em áreas de incisões prévias ou má realização da estomia, respectivamente ${ }^{18}$.

Nesses casos, o uso de placas planas e sistemas de duas peças são os mais indicados. Deve-se usar uma bolsa com tamanho suficiente para acomodar a alça e o efluente precedida da medição adequada do maior diâmetro da alça para que essa passe pela abertura sem traumas. $\mathrm{O}$ uso de um cinto pode ajudar a sustentar a bolsa e manter a placa apoiada ${ }^{1,17}$.

No relato dos cuidadores, evidenciou-se que as complicações em pele periestoma estão relacionadas ao tipo de bolsa e adesividade.

Às vezes não quer colar a bolsa, não quer segurar, não sei se porque sua muito. Não quer colar e solta, é muito trabalho. Nós vamos botando tudo que o povo manda e dá certo, porque ele faz é gastar bolsa. É brinquedo não! Um dia desse ele usou cola de sapateiro. (C1, C3, C4, $\mathrm{C} 6, \mathrm{C} 7, \mathrm{C} 8, \mathrm{C} 9)$.

O equipamento coletor indicado de forma inadequada, bem como a qualidade do material e o não uso de adjuvantes, compromete a integridade da pele periestoma. As características individuais de cada indivíduo, a localização da estomia e a técnica cirúrgica utilizada devem ser levadas 
em consideração durante a indicação do equipamento coletor e adjuvante ${ }^{19}$.

A maioria das pessoas com estomias intestinais tende a utilizar bolsas drenáveis, as quais podem ser esvaziadas periodicamente e trocadas entre 3 e 7 dias. No entanto, pessoas com trânsito intestinal regular, com evacuação a cada 2 dias, por exemplo, podem optar pelo uso de bolsa fechada com troca a cada eliminação ${ }^{1}$.

A aquisição das bolsas e demais insumos é garantida de forma gratuita mediante cadastro na associação dos ostomizados de cada município, conforme a avaliação da estomia e pele periestoma pelo enfermeiro do serviço.

A avaliação do enfermeiro estomaterapeuta nesse momento é de fundamental importância, visto que o cuidado de enfermagem exige do profissional a capacidade de agir e pensar criticamente acerca das informações obtidas na anamnese, dispondo, assim, de subsídios para refletir sobre processo de cuidado oferecido e sobre a qualidade da assistência com foco na reabilitação da pessoa com estomia intestinal ${ }^{18,20}$.

No tocante ao motivo pelo qual os participantes relacionam as complicações apresentadas em estomias e pele periestoma, os procedimentos cirúrgicos foram citados como causa principal:

O médico fez errado. No lugar dele deixar dois dedos para fora, ele fez reto, mais pra dentro que pra fora, então eu sofro com isso. Quando a bolsa cai, as fezes escorrem. Se fosse pra fora, mais uns dois dedos, não feria a barriga. (E12, E18)

O surgimento de complicações no pós-operatório onera as instituições de saúde, uma vez que, além de encarecerem o tratamento, aumentam o tempo de permanência hospitalar, comprometem o prognóstico e aumentam a carga de trabalho da equipe, prejudicando ainda mais a autoestima da pessoa com estomia intestinal e suas perspectivas de melhora.

Diante dos fatos, a incorporação de um enfermeiro estomaterapeuta no serviço, em especial naqueles que dispõem de poucos recursos, reduz grandemente as complicações em estomia e pele periestoma. Outra estratégia de redução dessas complicações é a demarcação préoperatória da estomia ${ }^{21}$.

Um estudo realizado com médicos cirurgiões no período de 2014 a 2015 revelou que a assistência prestada por esses profissionais a pessoas com estomias encontrava-se basicamente restrita à confecção da estomia e conduta clínica inicial, sendo necessário o encaminhamento dessas pessoas a serviços especializados a fim de garantir um acompanhamento contínuo após a alta hospitalar ${ }^{22}$.

Nesse contexto, a enfermagem em estomaterapia vem se destacando enquanto facilitadora do processo de cuidado, ocupando posição diferenciada entre os profissionais que prestam assistência a pessoas com estomias, pois, além de acompanhar todo o processo de confecção da estomia, atua prevenindo complicações e preparando para o processo de adaptação e autocuidado.

$\mathrm{O}$ uso inadequado das bolsas também foi citado pelos participantes cuidadores como a causa para o surgimento de dermatites na pele periestoma:

Eu acho que foi o líquido com ácido que queimou a pele, não sei se estava mal colocada a placa, a bolsa. (C2, C5)

A dermatite irritativa causada pelo contato do efluente com a pele periestoma leva ao surgimento de uma área hiperemiada, úmida e dolorosa. Isso acontece, muitas vezes, porque, ao cortar a bolsa, o orifício não é adequado ao tamanho da estomia. Nesses casos, a reavaliação quanto à técnica de autocuidado que está sendo utilizada é importante para melhorar a capacidade da pessoa com estomia intestinal ou seus cuidadores de providenciar um recorte mais adaptado a sua estomia ${ }^{14}$.

Há participantes que não atribuem causalidade para as complicações em estomia e pele periestoma:

Não sei não (risos). Eu não entendo dessas coisas não.(E3)

As orientações de autocuidado devem ser iniciadas a partir do momento em que houver indicação da confecção da estomia e podem perdurar até a completa reabilitação da pessoa com estomia. Tais orientações são de fundamental importância, uma vez que as estomias podem ser consideradas, por muitas pessoas, cirurgia mutiladora que causa transtornos do ponto de vista biopsicossocial e mudanças radicais em sua $Q V$, hábitos alimentares e higiênicos ${ }^{22}$.

Desse modo, o processo educativo não pode ser fragmentado, já que os sujeitos, imbuídos em multidimensionalidade, necessitam de cuidados holísticos e integrais. Assim, as orientações têm como objetivo ajudar a pessoa com estomia a superar as dificuldades de vida diária e conquistar a desejada inclusão social, a partir das mudanças que ocorrem em suas vidas decorrentes da estomia ${ }^{23}$. 


\section{CONCLUSÃO}

A partir da análise dos discursos dos participantes desta investigação, os significados atribuídos em relação às complicações da estomia e pele periestoma em um serviço de referência na região do Cariri foram relacionados ao tempo de permanência da bolsa, esforço físico, tipo de bolsa e adesividade, procedimentos cirúrgicos, uso inadequado das bolsas e outros que não atribuem causalidade para as complicações.

Diante do exposto, é possível observar que a ocorrência de complicações em estomia e pele periestoma é bastante comum entre os participantes entrevistados, seja devido a problemas relacionados ao manuseio e características dos equipamentos ou à presença de fatores de risco que podem predispor ao surgimento dessas complicações.

De forma geral, ficou evidenciado que tais complicações não estavam diretamente relacionadas à falta de informação do participante e seus cuidadores em relação aos cuidados com a estomia e pele periestoma, mas à forma como os profissionais de saúde estão gerindo esse processo.

É importante destacar a necessidade da realização de consultas de enfermagem focadas não só nas orientações quanto aos cuidados com a estomia e pele periestoma, mas na avaliação periódica que discuta, explique e demonstre as técnicas para o manuseio adequado dos equipamentos coletores conforme as característica individuais de cada participante (tipo de estomia, diâmetro, formato, altura, alergias, preferência pessoal, etc.), garantindo, dessa forma que essas informações sejam reproduzidas posteriormente.
A compreensão e as perspectivas do indivíduo acerca do processo de adaptação e possível reversão do caso influenciam significativamente na sua capacidade para o autocuidado. A confecção de uma estomia implica em um processo marcado pela dor, sofrimento físico e psicológico que atinge a equipe de saúde, o participante e seus familiares. Trata-se de um processo de adaptação lento que afeta, muitas vezes, pessoas em idade produtiva e traz repercussões negativas do ponto de vista afetivo, econômico, interpessoal e profissional.

Desse modo, a prestação de assistência especializada se faz necessária para que as orientações dos profissionais estejam direcionadas para as necessidades individuais de cada participante, a fim de se obter adesão ao autocuidado, reinserção social e retirada de dúvidas, uma vez que a assistência em saúde se encontra pautada em um processo interpessoal centrado no cuidado integral do indivíduo e sua família.

\section{CONTRIBUIÇÃO DOS AUTORES}

Conceitualização, Feitosa YS e Sampaio LRL; Metodologia, Feitosa YS; Sampaio LRL; Viana MCA; Sacramento KPN e Galdino YLS; Investigação, Feitosa YS; Carvalho TB; Silva FM; Moreira DAA e Mendonça FAC; Redação Primeira versão, Feitosa YS; Sampaio LRL; Carvalho TB e Moreira DAA; Redação - Revisão \& Edição, Sampaio LRL; Aquisição de Financiamento, Feitosa YS; Recursos, Feitosa YS; Supervisão, Sampaio LRL.

\section{REFERÊNCIAS}

1. Oliveira RG. Blackbook enfermagem. 1a ed. Belo Horizonte: Blackbook; 2016

2. Maurício VC, Souza NVDO, Costa CCP, Dias MO. The view of nurses about educational practices targeted at people with a stoma. Esc Anna Nery. 2017;21(4):21-4. https://doi. org/10.1590/2177-9465-EAN-2017-0003

3. Jayarajah U, Samarasekara AM, Samarasekera DN. A study of long-term complications associated with enteral ostomy and their contributory factors. BMC Res Notes. 2016;9(1):1-6. https://doi.org/10.1186/s13104-016-2304-z

4. Almeida EJ, Silva AL. Caracterização do perfil epidemiológico dos estomizados em hospitais da Secretaria de Estado de Saúde do Distrito Federal. ESTIMA, Braz J Enterostomal
Ther. 2015;13(1):11-6. $\quad$ https://doi.org/10.5327/Z18063144201500010004.

5. Instituto Nacional de Câncer. Incidência de câncer no Brasil [Internet]. Rio de Janeiro; 2018. [citado 20 Set 2018]. Disponível em: http://www.inca.gov.br/estimativa/2018/ sintese-de-resultados-comentarios.asp

6. Rocha JJR. Estomas intestinais (ileostomias e colostomias) e anastomoses intestinais. Medicina, Ribeirão Preto. 2011;44(1):51-6.

7. Dantas FG. Prevalência de complicações em pessoas com estomias urinárias e intestinais. Revista Enfermagem Atual. 2017;82(1):56-61.

8. Miguel VM, Jiménez EF, Parajó CA. Current status of the prevention and treatment of stoma complications. A 
narrative review. Cir Esp. 2014;92(3):149-56. https://doi. org/10.1016/j.ciresp.2013.09.011

9. Pengelly S, Reader J, Jones A, Roper K, Douie WJ, Lambert AW. Methods for siting emergency stomas in the absence of a stoma therapist. Ann R Coll Surg Engl. 2014;96(3):216-8. https://doi.org/10.1308/003588414X13814021679717

10. Forsmo HM, Pfeffer $F$, Rasdal A, Sintonen $H$, Körner $H$, Erichsen C. Pre and postoperative stoma education and guidance within an enhanced recovery after surgery (ERAS) programme reduces length of hospital stay in colorectal surgery. Int J Surg. 2016;36(1):121-6. https://doi. org/10.1016/j.ijsu.2016.10.031

11. Yamada BA, Ferrola EC, Azevedo GR, Blanes L, Rogenski NM, Santos VLCG. Estomaterapia - Competências do enfermeiro estomaterapeuta Ti Sobest ou do enfermeiro estomaterapeuta. Estima. 2008;6(1) [citado 12 Set 2018]. Disponível em: http://www.sobest.org.br/texto/11

12. Gil AC. Como elaborar projetos de pesquisa. 6a ed. São Paulo: Atlas; 2017.

13. Bardin L. Análise de conteúdo. 1a. ed. São Paulo: Edições 70; 2016.

14. Santos VLCG, Cesaretti IUR. Assistência em estomaterapia cuidando de pessoas com estomia. São Paulo: Atheneu; 2015.

15. Steinhagen E, Colwell J, Cannon L. Intestinal stomas Postoperative stoma care and peristomal skin complications. Clinics Colon Rectal Surgery. 2017;30(3):184-92. https://doi. org/10.1055/s-0037-1598159.

16. Secretaria de Atenção à Saúde. Portaria n .400 de 16 de novembro de 2009 [Internet]. Brasília, DF; 2009. Disponível em: http://www.ans.gov.br/images/stories/noticias/pdf/p sas 4002009 ostomizados.pdf

17. Miranda SM, Luz MHBA, Sonobe HM, Andrade EMLR, Moura ECC. Caracterização sociodemográfica e clínica de pessoas com estomia em Teresina. ESTIMA, Braz. J. Enterostomal Ther. 2016;14(1):29-35. https://doi.org/10.5327/Z18063144201600010005

18. Reis FF. Transição para a vivência com uma ostomia: intervenções de enfermagem [tese]. Porto: Universidade do Porto; 2015.

19. Collet JÁ, Silva FP, Aymone JLF. Bolsas coletoras utilizadas por estomizados: uma análise tridimensional. Design \& Tecnologia. 2016;11(1):1-10.

20. Moraes JT, Amaral CFS, Borges EL, Ribeiro MS, Guimarães EAA. Serviços de atenção ao estomizado: análise diagnóstica no estado de Minas Gerais, Brasil. Cad Saúde Colet. 2014;22(1):101-8. https://doi.org/10.1590/1414$\underline{462 \times 201400010015}$

21. Clarke L, Abbott $H$, Sharma P, Eglinton TW, Frizelle FA. Impact of restenting for recurrent colonic obstruction due to tumour ingrowth. BJS Open. 2017;1(6):202-6. https://doi. org/10.1002\%2Fbjs5.34

22. Moraes JT, Silva AE, Silva MDM, Guimarães RO, Ferraz GB. A percepção de cirurgiões sobre o cuidado em estomias. J Health Sci. 2017;19(1):14-8. https://doi.org/10.17921/24478938.2017v19n1p14-18

23. Lenza NFB, Sonobe HM, Buetto LS, Santos MG, Lima MS. O ensino do autocuidado aos pacientes estomizados e seus familiares: uma revisão integrativa. Rev Bras Prom Saúde. 2013;26(1):138-44. 